\title{
On Gamma Function Inequalities
}

\author{
By Joaquin Bustoz and Mourad E. H. Ismail*
}

To Jerry Fields, in friendship

\begin{abstract}
We show that certain functions involving quotients of gamma functions are completely monotonic. This leads to inequalities involving gamma functions. We also establish the infinite divisibility of several probability distributions whose Laplace transforms involve quotients of gamma functions.
\end{abstract}

1. Introduction. Motivated by Wallis's product

$$
\frac{\pi}{2}=\prod_{1}^{\infty}\left(\frac{2 n}{2 n-1}\right)\left(\frac{2 n}{2 n+1}\right)
$$

Kazarinoff [6] proved that the function $\theta(n)$,

$$
\frac{1 \cdot 3 \cdot 5 \cdots(2 n-1)}{2 \cdot 4 \cdot 6 \cdots(2 n)}=\frac{1}{\sqrt{\pi(n+\theta(n))}},
$$

satisfies

$$
\frac{1}{4}<\theta(n)<\frac{1}{2}, \quad n=1,2, \ldots
$$

More generally, set

$$
\theta(x)=-x+\left[\Gamma(x+1) / \Gamma\left(x+\frac{1}{2}\right)\right]^{2}, \quad x>-\frac{1}{2} .
$$

Watson [9] used Gauss' theorem [2, (14), p. 61] to obtain

$$
\theta(x)=-x+x_{2} F_{1}\left(-\frac{1}{2},-\frac{1}{2} ; x ; 1\right),
$$

that is,

$$
\theta(x)=\sum_{m=1}^{\infty} \frac{\left(-\frac{1}{2}\right)_{m}\left(-\frac{1}{2}\right)_{m}}{m !(x+1)_{m-1}},
$$

with the usual notation $(\sigma)_{n}=\Gamma(\sigma+n) / \Gamma(\sigma)$, that is,

$$
(\sigma)_{0}=1, \quad(\sigma)_{m}=\sigma(\sigma+1) \cdots(\sigma+m-1), \quad m>0 .
$$

Watson then observed that (1.4) implies that $\theta(x)$ is decreasing for $x>-\frac{1}{2}$ and $\theta(\infty)=\frac{1}{4}$ and $\theta\left(-\frac{1}{2}\right)=\frac{1}{2}$. This obviously implies the sharper inequalities

$$
\frac{1}{4}<\theta(x) \leqslant \frac{1}{2}, \quad x \geqslant-\frac{1}{2} .
$$

Received September 30, 1985; revised December 31, 1985.

1980 Mathematics Subject Classification. Primary 33A15, 26 D20.

Key words and phrases. Gamma function, psi function, complete monotonicity, inequalities, infinite divisibility, Laplace transform.

* Partially supported by NSF grant DMS 8503731. 
For related inequalities and further references we refer the interested reader to [5] and [7].

More recently Dutka [1] proved

$$
\begin{gathered}
(1+1 / 2 n)^{1 / 2}-1<\theta(n) / n<(1-1 / 2 n)^{-1 / 2}-1, \quad n=1,2, \ldots, \\
\frac{1}{4}<\theta(n)<(n+1) /(4 n+3), \quad n=1,2, \ldots
\end{gathered}
$$

The methods used to prove (1.5), (1.6) and (1.7) are not systematic and do not seem to explain why inequalities of this type should exist. Recall the definition of completely monotonic functions.

Definition. A function $f(x)$ is said to be completely monotonic on an interval $I$ if $(-1)^{n} f^{(n)}(x) \geqslant 0$ on $I$.

Completely monotonic functions are useful in many fields, including mathematical analysis [10], probability theory [3] and numerical analysis [11].

The purpose of this work is to point out that inequalities like (1.5), (1.6), or (1.7) are immediate consequences of the complete monotonicity of certain functions. Indeed, one should investigate monotonicity properties of functions involving quotients of gamma functions and as a by-product derive inequalities of the aforementioned type. This approach is simpler and yields more general results. Watson's representation (1.4) gave us the clue, because (1.4) shows that $\theta(x)$ is completely monotonic on $\left[-\frac{1}{2}, \infty\right)$. In Section 2 we shall prove

THEOREM 1. Let

$$
f(x):=(x+c)^{-1 / 2} \Gamma(x+1) / \Gamma\left(x+\frac{1}{2}\right), \quad x>\max \left(-\frac{1}{2},-c\right) .
$$

Then

(i) $f(x)$ is completely monotonic on $(-c, \infty)$ if $c \leqslant \frac{1}{4}$;

(ii) $1 / f(x)$ is completely monotonic on $\left[-\frac{1}{2}, \infty\right)$ if $c \geqslant \frac{1}{2}$.

An immediate corollary is

Corollary 2. The function $f(x)$ is increasing (decreasing) on $\left[-\frac{1}{2}, \infty\right)$ (on $(-c, \infty))$ if $c \geqslant \frac{1}{2}\left(c \leqslant \frac{1}{4}\right)$.

We shall see in Section 4 that Corollary 2 implies (1.5) and (1.7). In Section 2 we shall also prove the following generalization of Theorem 1 .

THEOREM 3. Let

$$
a+1 \geqslant b>a, \quad \alpha:=\max (-a,-c), \quad \beta:=\max (-b,-c)
$$

and

$$
g(x ; a, b, c)=(x+c)^{a-b} \Gamma(x+b) / \Gamma(x+a), \quad x>\alpha .
$$

Then

(i) $g(x ; a, b, c)$ is completely monotonic on $(\alpha, \infty)$ if $c \leqslant \frac{1}{2}(a+b-1)$;

(ii) $1 / g(x ; a, b, c)$ is completely monotonic for $x>\beta$ if $c \geqslant a$.

We now consider inequalities of the type (1.6). Such inequalities really involve the function $[n+\theta(n)] / n$, i.e.,

$$
\Gamma(x) \Gamma(x+1) / \Gamma^{2}\left(x+\frac{1}{2}\right), \quad x=1,2,3, \ldots
$$

In Section 3 we shall prove Theorems 4, 5 and 6. 
THEOREM 4. The function

$$
\left(1-\frac{1}{2 x}\right)^{-1 / 2} \frac{\Gamma^{2}(x+1 / 2)}{\Gamma(x) \Gamma(x+1)}
$$

is completely monotonic on $\left(\frac{1}{2}, \infty\right)$.

THEOREM 5. The function

$$
(1+1 / 2 x)^{-1 / 2} \Gamma(x) \Gamma(x+1) / \Gamma^{2}(x+1 / 2)
$$

is completely monotonic on $(0, \infty)$.

THEOREM 6. The function

$$
p(x ; a, b)=\frac{\Gamma(x) \Gamma(x+a+b)}{\Gamma(x+a) \Gamma(x+b)}, \quad a, b \geqslant 0
$$

is completely monotonic on $(0, \infty)$.

Observe that

$$
(\theta(x)+x) / x=p\left(x, \frac{1}{2}, \frac{1}{2}\right),
$$

so Watson's result is a special case of Theorem 6, as we shall see in Section 4.

Gautschi [4] proved that if $0<s<1, x \geqslant 1$, then

$$
x^{1-s}<\Gamma(x+1) / \Gamma(x+s)<\exp [(1-s) \psi(x+(s+1) / 2)],
$$

$\psi(z):=\Gamma^{\prime}(z) / \Gamma(z)$. Recently, Kershaw [8] established the closer bounds

$$
\begin{gathered}
\exp \left[(1-s) \psi\left(x+s^{1 / 2}\right)\right]<\frac{\Gamma(x+1)}{\Gamma(x+s)}<\exp \left[(1-s) \psi\left(x+\frac{s+1}{2}\right)\right] \\
\left(x+\frac{s}{2}\right)^{1-s}<\frac{\Gamma(x+1)}{\Gamma(x+s)}<\left[x-\frac{1}{2}+\left(s+\frac{1}{4}\right)^{1 / 2}\right]^{1-s}
\end{gathered}
$$

for $x>0$ and $0<s<1$.

In Section 3, we shall prove the following more general results.

THEOREM 7. The functions

$$
\begin{gathered}
\frac{\Gamma(x+s)}{\Gamma(x+1)} \exp \left[(1-s) \psi\left(x+\frac{s+1}{2}\right)\right], \\
\Gamma(x+1)(x+s / 2)^{s-1} / \Gamma(x+s)
\end{gathered}
$$

are completely monotonic on $(0, \infty)$ for $0 \leqslant s \leqslant 1$. When $0<s<1$, the functions (1.17), (1.18) satisfy $(-1)^{n} f^{(n)}(x)>0, x>0$.

TheOREM 8. Let $0<s<1$ and $x>0$. Then both

$$
\frac{\Gamma(x+1)}{\Gamma(x+s)} \exp \left[(s-1) \psi\left(x+s^{1 / 2}\right)\right],
$$

and

$$
\Gamma(x+s)\left[x-\frac{1}{2}+\left(s+\frac{1}{4}\right)^{1 / 2}\right]^{1-s} / \Gamma(x+1)
$$

are strictly decreasing functions.

In Section 4 we shall show how Theorems 7 and 8 generalize (1.15) and (1.16). We shall also discuss related inequalities and infinitely divisible probability distributions. 
2. Proofs of Theorems 1 and 3. Our proofs rely on the following well-known lemma which can be proved by successive applications of the chain rule.

LEMMA 2.1. The function $\exp (-h(x))$ is completely monotonic on an interval $I$ if $h^{\prime}(x)$ is completely monotonic on $I$.

Proof of Theorem 1. First consider the case $c \leqslant \frac{1}{4}$. In this case let

$$
h(x):=-\ln f(x) \text {, }
$$

hence

$$
h^{\prime}(x)=\frac{1}{2 x+2 c}+\frac{\Gamma^{\prime}\left(x+\frac{1}{2}\right)}{\Gamma\left(x+\frac{1}{2}\right)}-\frac{\Gamma^{\prime}(x+1)}{\Gamma(x+1)} .
$$

By the above lemma it suffices to show that $h^{\prime}(x)$ is completely monotonic. Recall that $\psi(z):=\Gamma^{\prime}(z) / \Gamma(z)$. The integral representation

$$
\psi\left(\frac{1}{2}+\frac{1}{2} z\right)-\psi\left(\frac{1}{2} z\right)=2 \int_{0}^{\infty} e^{-z t}\left(1+e^{-t}\right)^{-1} d t, \quad \operatorname{Re} z>0
$$

(see (1) and (3) on page 20 and (1) on page 15 of [2]), gives

$$
h^{\prime}(x)=\int_{0}^{\infty} e^{-2(x+c) t} d t-2 \int_{0}^{\infty} e^{-(2 x+1) t}\left(1+e^{-t}\right)^{-1} d t
$$

that is,

$$
h^{\prime}(x)=\int_{0}^{\infty} \frac{e^{-2(x+c) t}}{1+e^{-t}}\left[\left(1-e^{-t / 2}\right)^{2}+2 e^{-t / 2}\left(1-e^{2(c-1 / 4) t}\right)\right] d t .
$$

This implies the complete monotonicity of $h^{\prime}(x)$ when $c \leqslant \frac{1}{4}$, since the integrand is $e^{-2 x t}$ times a nonnegative function of $t$.

When $c \geqslant \frac{1}{2}$, we rewrite $(2.4)$ in this form,

$$
-h^{\prime}(x)=\int_{0}^{\infty} \frac{e^{-2 x t}}{1+e^{-t}}\left[2 e^{-t}-e^{-2 c t}-e^{-(2 c+1) t}\right] d t .
$$

The integrand in the above equation is $e^{-2 x t}$ times a nonnegative function of $t$, hence $-h^{\prime}(x)$ is completely monotonic. This establishes part (ii).

Proof of Theorem 3. Since $(1-\mu / x)^{-\lambda}$ is completely monotonic on $(0, \infty)$ for $\lambda>0, \mu>0$, and the product of completely monotonic functions is also completely monotonic, it suffices to consider the case $c=(a+b-1) / 2$. Part (i) will follow if we can show that $-\ln g$ has a completely monotonic derivative on the interval under consideration. Set

$$
\xi(x):=-\ln g(x ; a, b,(a+b-1) / 2) .
$$

Clearly,

$$
\xi^{\prime}(x)=\frac{2(b-a)}{2 x+a+b-1}+\frac{\Gamma^{\prime}(x+a)}{\Gamma(x+a)}-\frac{\Gamma^{\prime}(x+b)}{\Gamma(x+b)} .
$$

We now apply the integral representation, [2, (14), p. 16],

$$
\Gamma^{\prime}(z) / \Gamma(z)=-\gamma+\int_{0}^{\infty}\left(e^{-t}-e^{-t z}\right)\left(1-e^{-t}\right)^{-1} d t, \quad \operatorname{Re} z>0
$$


$\gamma$ being Euler's constant. This gives

$$
\begin{aligned}
\xi^{\prime}(x) & =(b-a) \int_{0}^{\infty} e^{-[x+(a+b-1) / 2] t}+\int_{0}^{\infty} \frac{e^{-x t}}{1-e^{-t}}\left\{e^{-b t}-e^{-a t}\right\} d t \\
& =\int_{0}^{\infty} \frac{\omega(t)}{1-e^{-t}} \exp [-t\{x+(a+b) / 2\}] d t
\end{aligned}
$$

with $\omega(t)=2(b-a) \sinh (t / 2)-2 \sinh [(b-a) t / 2]$. Clearly,

$$
\frac{1}{2} \omega(2 t)=\sum_{n=0}^{\infty}(b-a) \frac{t^{2 n+1}}{(2 n+1) !}\left\{1-(b-a)^{2 n-1}\right\}
$$

shows that $\omega(t) \geqslant 0$ for $t \geqslant 0$, hence $\xi^{\prime}(x)$ is completely monotonic on $(\alpha, \infty)$. This establishes part (i). We now proceed with proving part (ii). Using the integral representation (2.6), one can easily derive

$$
\frac{d}{d x} \ln g(x ; a, b, c)=\int_{0}^{\infty} \omega(t) \exp [-x(t+c)] d t
$$

with $\omega(t)=(a-b)\left(1-e^{-t}\right)+e^{(c-a) t}-e^{(c-b) t}$. Since $\omega(t)$ is an increasing function of $c$, it suffices to prove the positivity of $\omega(t)$ when $c=a$. If $c=a$, we have

$$
\omega^{\prime}(t)=(a-b) e^{-t}-(a-b) e^{(a-b) t}, \quad c=a, t>0,
$$

which is clearly nonnegative for $t \geqslant 0$. Thus $\omega(t)$ is nondecreasing on $(0, \infty)$. On the other hand, $\omega(0)=0$. Therefore $\omega(t) \geqslant 0$ for $t \geqslant 0$ and $c \geqslant a$, which implies the complete monotonicity of $\ln g(x ; a, b, c)$. Finally, part (ii) follows from the lemma mentioned at the beginning of this section.

3. Proofs of Theorems 4-8. We start by proving Theorem 4.

Proof of Theorem 4. Let $-h(x)$ denote the logarithm of the function (1.11). Therefore,

$$
h^{\prime}(x)=\frac{\Gamma^{\prime}(x)}{\Gamma(x)}+\frac{\Gamma^{\prime}(x+1)}{\Gamma(x+1)}-\frac{2 \Gamma^{\prime}\left(x+\frac{1}{2}\right)}{\Gamma\left(x+\frac{1}{2}\right)}-\frac{1}{2}\left(\frac{1}{x-\frac{1}{2}}-\frac{1}{x}\right),
$$

and using (2.3) we obtain the integral representation

$$
\begin{aligned}
h^{\prime}(x) & =2 \int_{0}^{\infty} \frac{e^{-2 x t}}{1+e^{-t}}\left(e^{-t}-1\right)+\int_{0}^{\infty}\left[e^{-(2 x-1) t}-e^{-2 x t}\right] d t \\
& =\int_{0}^{\infty} \frac{e^{-2 x t}}{1+e^{-t}}\left[2\left(e^{-t}-1\right)+\left(e^{t}-1\right)\left(1+e^{-t}\right)\right] d t \\
& =\int_{0}^{\infty} e^{-2 x t} \frac{\left(e^{t}-1\right)}{1+e^{-t}}\left(1-e^{-t}\right) d t
\end{aligned}
$$

This proves the complete monotonicity of $h^{\prime}(x)$ on $\left(\frac{1}{2}, \infty\right)$, hence $e^{-h(x)}$ is also completely monotonic on $\left(\frac{1}{2}, \infty\right)$. 
Proof of Theorem 5. As in the above proof, let the function in (1.12) be $e^{-w(x)}$. Similarly, we derive

$$
\begin{aligned}
w^{\prime}(x) & =2 \int_{0}^{\infty} \frac{e^{-2 x t}}{1+e^{-t}}\left(1-e^{-t}\right)+\frac{1}{2 x+1}-\frac{1}{2 x} \\
& =2 \int_{0}^{\infty} \frac{e^{-2 x t}}{1+e^{-t}}\left(1-e^{-t}\right)+\int_{0}^{\infty} e^{-2 x t}\left(e^{-t}-1\right) d t \\
& =\int_{0}^{\infty} e^{-2 x t} \frac{\left(1-e^{-t}\right)^{2}}{1+e^{-t}} d t
\end{aligned}
$$

which establishes Theorem 5 .

Proof of Theorem 6. Let $q(x ; a, b)=-\ln p(x ; a, b)$. Applying (2.6) we obtain

$$
q^{\prime}(x ; a, b)=\int_{0}^{\infty} \frac{e^{-t x}}{\left(1-e^{-t}\right)}\left(1-e^{-a t}\right)\left(1-e^{-b t}\right) d t,
$$

which implies the complete monotonicity of $q^{\prime}(x ; a, b)$ on $(0, \infty)$. Finally, Lemma 2.1 establishes the complete monotonicity of $p(x ; a, b)$ on $(0, \infty)$ and the proof is complete.

Our proof of Theorem 7 relies on

Lemma 3.1. Let $0<a<b$ and $y>0$. Then

$$
(y+a)^{-n}-(y+b)^{-n}>(b-a) n[y+(a+b) / 2]^{-n-1}, \quad n>0 .
$$

Proof. Let $c=(a+b) / 2, d=(b-a) / 2$, so $a=c-d, b=c+d$. Clearly $0<d / c<1$, and the binomial theorem yields

$$
\begin{aligned}
(y+a)^{-n}-(y+b)^{-n} & =(y+c)^{-n} \sum_{k=0}^{\infty} \frac{(n)_{k}}{k !}\left(\frac{d}{y+c}\right)^{k}\left[1-(-1)^{k}\right] \\
& >2 n d(y+c)^{-n-1} .
\end{aligned}
$$

We now prove Theorem 7.

Proof of Theorem 7. Let the functions in (1.17) and (1.18) be $\exp \left(-h_{j}(x)\right)$, $j=1,2$, respectively. It is clear that

$$
h_{1}^{\prime}(x)=\psi(x+1)-\psi(x+s)+(s-1) \psi^{\prime}(x+(s+1) / 2) .
$$

Using [2, p. 15],

$$
\psi(x)=-\gamma-\frac{1}{x}+\sum_{n=1}^{\infty}\left[\frac{1}{n}-\frac{1}{x+n}\right]
$$

we obtain

Therefore

$$
h_{1}^{\prime}(x)=\sum_{n=0}^{\infty}\left[\frac{1}{x+n+s}-\frac{1}{x+n+1}+\frac{s-1}{[x+n+(s+1) / 2]^{2}}\right] .
$$

$$
\begin{aligned}
& \frac{(-1)^{n}}{n !} h_{1}^{(n+1)}(x) \\
& =\sum_{k=0}^{\infty}\left[(x+k+s)^{-n-1}-(x+k+1)^{-n-1}\right. \\
& \left.+(n+1)(s-1)\{x+k+(s+1) / 2\}^{-n-2}\right],
\end{aligned}
$$


and the positivity of $(-1)^{n} h_{1}^{(n+1)}(x)$ on $(0, \infty), 0<s<1$, follows from Lemma 3.1. Similarly,

$$
\begin{aligned}
h_{2}^{\prime}(x) & =\frac{1-s}{x+s / 2}+\sum_{k=0}^{\infty}\left\{\frac{1}{x+k+1}-\frac{1}{x+k+s}\right\} \\
& =\int_{0}^{\infty} e^{-x t}\left[(1-s) e^{-s t / 2}+\left(e^{-t}-e^{-s t}\right) /\left(1-e^{-t}\right)\right] d t \\
& =\int_{0}^{\infty} e^{-(x+s / 2) t}\left[1-s-\frac{\sinh \{(1-s) t / 2\}}{\sinh (t / 2)}\right] d t
\end{aligned}
$$

The complete monotonicity of $h_{2}^{\prime}(x)$ now follows from the positivity of

$$
(1-s) \sinh (t)-\sinh (1-s) t
$$

on $(0, \infty)$ for $0<s<1$.

Proof of Theorem 8. Let $h_{3}(x)$ and $h_{4}(x)$ denote logarithms of the functions in (1.19) and (1.20), respectively. The series representation (3.1) gives

$$
\begin{aligned}
h_{3}^{\prime}(x) & =\sum_{k=0}^{\infty}\left[\frac{1}{x+s+k}-\frac{1}{x+1+k}+\frac{(s-1)}{(x+k+\sqrt{s})^{2}}\right] \\
& =(1-s) \sum_{k=0}^{\infty}\left[\frac{1}{(x+1+k)(x+s+k)}-\frac{1}{(x+k+\sqrt{s})^{2}}\right] .
\end{aligned}
$$

But $(X+\sqrt{s})^{2}-(X+1)(X+s)=-X(1-\sqrt{s})^{2}$, which is negative for $0<s<1$.

This shows that $h_{3}^{\prime}(x)<0$. Similarly, one can show that

$$
\begin{aligned}
& h_{4}^{\prime}(x) /(1-s)=\frac{1}{x-\frac{1}{2}+\sqrt{s+\frac{1}{4}}}-\sum_{k=0}^{\infty} \frac{1}{(x+1+k)(x+2+k)} \\
& =\sum_{k=0}^{\infty}\left[\frac{1}{x+k-\frac{1}{2}+\sqrt{s+\frac{1}{4}}}-\frac{1}{x+k+\frac{1}{2}+\sqrt{s+\frac{1}{4}}} \frac{1}{(x+1+k)(x+s+k)}\right] .
\end{aligned}
$$

Observe that

$$
\begin{aligned}
(X+s)(X+1) & -\left(X-\frac{1}{2}+\sqrt{s+\frac{1}{4}}\right)\left(X+\frac{1}{2}+\sqrt{s+\frac{1}{4}}\right) \\
& =X\left(s+1-2 \sqrt{s+\frac{1}{4}}\right)<0 \text { for } X>0,0<s<1
\end{aligned}
$$

This proves the negativity of $h_{4}^{\prime}(x)$ and the proof is complete.

4. Related Inequalities and Infinitely Divisible Distribution. Recall that [2, (4), p. 49]

$$
\Gamma(x+a) / \Gamma(x+b) \approx x^{a-b} \quad \text { as } x \rightarrow \infty .
$$

Therefore $f(x) \rightarrow 1$ as $x \rightarrow \infty$, where $f$ is as in (1.8). Corollary 2 gives

$$
\begin{aligned}
& f(x)>1 \text { for } x>-c \text { when } c \leqslant \frac{1}{4}, \\
& f(x)<1 \text { for } x>-\frac{1}{2} \text { when } c \geqslant \frac{1}{2} .
\end{aligned}
$$

In particular,

$$
\sqrt{x+\frac{1}{2}}>\frac{\Gamma(x+1)}{\Gamma\left(x+\frac{1}{2}\right)}>\sqrt{x+\frac{1}{4}}, \quad x>-\frac{1}{4} .
$$

The inequalities (4.2) are the same as (1.5). 
The inequalities (1.6) similarly follow from Theorems 4 and 5 and the asymptotic relationship (4.1). This actually proves the following stronger version of (1.6),

$$
(1+1 / 2 x)^{1 / 2}<1+\theta(x) / x<(1-1 / 2 x)^{-1 / 2}, \quad x>\frac{1}{2} .
$$

The inequalities (1.7) follow easily from (4.2) and (4.3), again with $n$ treated as a continuous parameter greater than $\frac{1}{2}$.

We now explore implications of Theorems 6, 7, 8. The asymptotic relationship (4.1) shows that $\lim _{x \rightarrow \infty} p(x ; a, b)=1$. Therefore, in view of Theorem 6 , we get

$$
1<\frac{\Gamma(x) \Gamma(x+a+b)}{\Gamma(x+a) \Gamma(x+b)}, \quad x>0, a, b \geqslant 0 .
$$

This is a generalization of (1.4), because

$$
\frac{\Gamma(x) \Gamma(x+a+b)}{\Gamma(x+a) \Gamma(x+b)}={ }_{2} F_{1}(-a,-b ; x ; 1), \quad \operatorname{Re} x>-a-b .
$$

The above ${ }_{2} F_{1}$ is obviously completely monotonic if there is a nonnegative integer $n$ such that $n+1>a, b>n$ or if $\min (a, b)=n$. In all other cases, Theorem 6 gives the complete monotonicity of the functions appearing in (4.5) and provides the generalization alluded to in Section 1. On the other hand, the asymptotic formulas (4.1) and [2, p. 47],

$$
\psi(x) \approx \ln x \text { as } x \rightarrow \infty,
$$

show that as $x \rightarrow \infty$ the functions (1.17)-(1.20) tend to 1 . This and Theorems 7 and 8 give Kershaw's inequalities (1.15) and (1.16).

Finally, we discuss related infinitely divisible distributions.

Definition. A probability measure $d \mu$ is infinitely divisible if for every positive integer $n$ there exists a probability measure $d \mu_{n}$ such that

$$
d \mu=\underbrace{d \mu_{n} * d \mu_{n} * \cdots * d \mu_{n}}_{n \text {-times }},
$$

where * denotes convolution.

The connection between infinitely divisible probability measures supported on $(0, \infty)$ and completely monotonic functions is the following, Feller [3].

LEMma 4.1. A probability measure $d \mu$ with $\operatorname{supp} d \mu \subset(0, \infty)$ is infinitely divisible if and only if

$$
\int_{0}^{\infty} e^{-x t} d \mu(t)=e^{-h(x)}, \quad x \geqslant 0,
$$

with $h(0)=0$ and $h^{\prime}(x)$ completely monotonic on $(0, \infty)$.

Lemma 4.1 and the proofs of Theorems 3, 4, 5, 6, and 7 establish Theorem 4.2 below.

THEOREM 4.2. The following functions are Laplace transforms of infinitely divisible probability distributions:

(i) $g(x+\alpha+\varepsilon ; a, b, c) / g(\alpha+\varepsilon ; a, b, c)$, if $\varepsilon>0,2 c \leqslant a+b-1$ and (1.9) holds;

(ii) $g(x+\beta+\varepsilon ; a, b, c) / g(\beta+\varepsilon ; a, b, c)$, if $\varepsilon>0, c \geqslant a$ and (1.9) holds; 
(iii) $w_{1}(x+\varepsilon) / w_{1}(\varepsilon)$, if $\varepsilon>0$, where

$$
w_{1}(x)=\left[1-(2 x+1)^{-1}\right]^{-1 / 2} \Gamma^{2}(x+1) /\left[\Gamma\left(x+\frac{1}{2}\right) \Gamma\left(x+\frac{3}{2}\right)\right] ;
$$

(iv) $w_{2}(x+\varepsilon) / w_{2}(\varepsilon)$, if $\varepsilon>0$ and

$$
w_{2}(x)=[1+1 /(2 x)]^{-1 / 2} \Gamma(x) \Gamma(x+1) / \Gamma^{2}(x+1 / 2) ;
$$

(v) $p(x+\varepsilon ; a, b) / p(\varepsilon ; a, b), a, b, \varepsilon>0$;

(vi) $w_{3}(x+\varepsilon) / w_{3}(\varepsilon), \varepsilon>0,0 \leqslant s \leqslant 1$, where $w_{3}(x)$ is the function in (1.17);

(vii) $w_{4}(x+\varepsilon) / w_{4}(\varepsilon)$, for $\varepsilon>0,0 \leqslant s \leqslant 1$, and $w_{4}(x)$ denotes the function in (1.18).

Acknowledgments. The authors thank Waleed Al-Salam and Jerry Fields of the University of Alberta for their critical comments on an earlier draft of this paper.

Department of Mathematics

Arizona State University

Tempe. Arizona 85287

1. J. DutKa, “On some gamma function inequalities," SIAM J. Math. Anal., v. 16, 1985, pp. 180-185.

2. A. Erdelyi, W. Magnus, F. Oberhettinger \& F. G. Tricomi, Higher Transcendental Functions, Vol. 1, McGraw-Hill, New York, 1953.

3. W. Feller, An Introduction to Probability Theory and its Applications, Wiley, New York, 1966.

4. W. GautsChI, "Some elementary inequalities relating to the gamma and incomplete gamma function," J. Math. Phys., v. 38, 1959, pp. 77-81.

5. M. E. H. Ismail, L. Lorch \& M. Muldoon, "Completely monotonic functions associated with the gamma function and its $q$-analogues," J. Math. Anal. Appl., v. 116, 1986, pp. 1-9.

6. D. K. Kazarinoff, “On Wallis' formula,” Edinburgh. Math. Soc. Notes, No. 40, 1956, pp. 19-21.

7. A. KeMP, “On gamma function inequalities,” Skand. Aktuarietidskr., v. 56, 1973, pp. 65-69.

8. D. Kershaw, "Some extensions of W. Gautschi's inequalities for the gamma function," Math. Comp., v. 41, 1983, pp 607-611.

9. G. N. Watson, "A note on gamma functions," Proc. Edinhurgh Math. Soc. (2), v. 11, 1958/1959. Edinburgh Math. Soc. Notes, No. 42, 1959, pp. 7-9.

10. D. V. Widder, The Laplace Transform, Princeton Univ. Press, Princeton, N.J., 1944.

11. J. WIMP, Sequence Transformations and Their Applications, Academic Press, New York, 1981. 